\title{
Effectiveness of interventions targeting physical activity, nutrition and healthy weight for university and college students: a systematic review and meta-analysis
}

\author{
Ronald C Plotnikoff ${ }^{1,2^{*}}$, Sarah A Costigan ${ }^{1,2}$, Rebecca L Williams ${ }^{1,3}$, Melinda J Hutchesson ${ }^{1,3}$, Sarah G Kennedy ${ }^{1,2}$, \\ Sara L Robards ${ }^{1,2}$, Jennifer Allen², Clare E Collins ${ }^{1,3}$, Robin Callister ${ }^{1,4}$ and John Germov ${ }^{5}$
}

\begin{abstract}
To examine the effectiveness of interventions aimed at improving physical activity, diet, and/or weight-related behaviors amongst university/college students. Five online databases were searched (January 1970 to April 2014). Experimental study designs were eligible for inclusion. Data extraction was performed by one reviewer using a standardized form developed by the researchers and checked by a second reviewer. Data were described in a narrative synthesis and meta-analyses were conducted when appropriate. Study quality was also established. Forty-one studies were included; of these, 34 reported significant improvements in one of the key outcomes. Of the studies examining physical activity 18/29 yielded significant results, with meta-analysis demonstrating significant increases in moderate physical activity in intervention groups compared to control. Of the studies examining nutrition, 12/24 reported significantly improved outcomes; only 4/12 assessing weight loss outcomes found significant weight reduction. This appears to be the first systematic review of physical activity, diet and weight loss interventions targeting university and college students. Tertiary institutions are appropriate settings for implementing and evaluating lifestyle interventions, however more research is needed to improve such strategies.
\end{abstract}

Keywords: University, College, Tertiary education institutions, University students, Health promotion, Health behavior, Healthy universities, Physical activity, Exercise, Diet, Nutrition, Weight loss

\section{Introduction}

Physical inactivity and poor dietary-intake are related behaviors that impact on health and wellbeing and the maintenance of a healthy weight. These behaviors underpin risk of lifestyle related non-communicable conditions [1]. Risk for ischaemic heart disease, stroke, type two diabetes, osteoporosis, various cancers and depression are linked by behavioral and biomedical health determinants such as physical inactivity, poor dietary behaviors and overweight/obesity [2].

\footnotetext{
* Correspondence: Ron.plotnikoff@newcastle.edu.au

'Priority Research Centre for Physical Activity and Nutrition, University of Newcastle, Callaghan Campus, Newcastle, NSW, Australia

${ }^{2}$ School of Education, Faculty of Education and Arts, University of Newcastle, Callaghan Campus, Newcastle, NSW, Australia

Full list of author information is available at the end of the article
}

The health benefits of engaging in regular physical activity are well established for adults [3]. Strategies to promote physical activity have become an important public health approach for the prevention of chronic diseases [4]. The prevalence of achieving physical activity recommendations declines rapidly between the ages of 18 and 24 [5] when many young people are undertaking tertiary education [6-8]. For instance, in the United States nearly half of all university students are not achieving recommended levels of physical activity [9]. Australian data in the $\geq 18$ year age group indicate $66.9 \%$ are sedentary or have low levels of physical activity during 2011-2012 [10]. Similarly in the United Kingdom, $73 \%$ of male and $79 \%$ of female university students do not meet physical activity guidelines [6]. Further, Irwin [8] suggests that students living on campus are less likely to be active, and thus may be at greater risk of poor health. 
Dietary intake patterns that align with national dietary guidelines are associated with reduced risk of developing chronic conditions $[11,12]$, however recent research suggests tertiary students do not achieve these guidelines [13-15]. For instance, in the United States, university and college students have sub-optimal dietary habits compared to such recommendations [16]. Similarly, Australian tertiary students fail to consume the recommended daily servings of fruit (50\%) and vegetables (90\%) [2]. While students from the UK fail to consume the recommended daily intake of fruit and vegetables $(88.7 \%$ and $83.5 \%$, respectively) [17].

Commencing college/university is often associated with students having more autonomy over their dietary choices (e.g., food purchasing and preparation). Due to life stage, students may not consider the risk of developing chronic diseases when making food choices [18]. Specifically, factors such as cost, skipping meals, inadequate variety of foods, snacking, and frequent consumption of fast foods may increase students' risk of poor health [19]. Indeed, studies have reported that considerable weight gain occurs during college/university [20,21]. The associated food selection skills and habits have long-term health impacts [22]. Further, within US institutions a great proportion of freshmen (first year) live in college resident halls, which provide commercially prepared food, take-away and preprepared meals. This environment may further contribute to subsequent poor food purchasing and preparation behaviors. Along with these dietary behaviors, physical activity participation also declines in university and college students, which may be due to increased sedentary time when studying and during examination periods [23].

Given the lack of physical activity and healthy eating it is not surprising that the prevalence of overweight/obesity has reached epidemic proportions in young adults. In the USA, the age range of greatest increase in obesity $(7.1 \%$ to $12.1 \%$ ) is among young adults aged $18-29$ years [7]. Indeed, late adolescence and early adulthood appear to be significant periods of transition, highlighting the importance of understanding factors such as attitudes towards and knowledge of health benefits, as these may be associated with physical activity levels, dietary behavior and obesity prevalence [24]. Improvements to lifestyle behaviors can reduce or prevent the occurrence of non-communicable diseases; therefore strategies to foster healthier lifestyles in the working age population are essential.

Higher education institutions are an appropriate setting to promote healthy lifestyles. First, universities and colleges have the potential to engage large numbers of students in behavior change interventions, and the estimated number of individuals participating in higher education is continuing to rise [25]. It is projected that student numbers in American colleges will reach 22 million in 2014, and that the number of students enrolled in higher education worldwide will reach 262 million by 2025 , a marked increase from 178 million in 2010 [26]. Second, higher education institutions have access to a large proportion of students living away from home for the first time, and have the capacity to provide support and establish healthy behavioral patterns that may continue throughout the lifespan. Third, universities and colleges are regarded as organizations that follow high standards of practice which can establish research-based examples for surrounding communities to follow. This allows for the opportunity and responsibility to develop and implement the best available research evidence, and to set a benchmark for other groups to follow [27]. Universities and colleges have a range of facilities, resources and qualified staff, commonly including health professionals, ideal for implementing initiatives to target lifestyle-related health issues. Finally, the possibility that exists for students to deliver initiatives as a part of their study/training to become health professionals adds to the promise for tertiary education institutions as ideal settings for promoting healthy lifestyles.

Evidence suggests that intervention strategies have been successful for students in the higher educational setting [1,5], particularly interventions that seek to empower individuals to achieve their full potential through creating learning and support to improve health, wellbeing and sustainability within the community [28]. In addition, whilst the primary advantage of implementing health promotion programs is to reduce individuals' health risks, the benefits to higher education institutions in attrition, retention and academic performance are also potential gains [29]. Although a recent review examining the effectiveness of interventions targeting health behaviors (physical activity, nutrition and healthy weight) amongst university staff has been conducted [22], it appears that a review investigating the effectiveness of health behavior interventions on these health behaviors/issues for university students has not yet been performed.

\section{Objective}

The objective of this paper is to systematically review the best available evidence regarding the impact of health behavior interventions to improve physical activity, diet and/ or weight outcomes and targeted at students enrolled in tertiary education institutions.

\section{Review \\ Methods}

Data source

A structured electronic search employing PRISMA reporting guidelines [30] was performed on health-focused intervention studies carried out in tertiary level educational institutions and published between January 1970 and April 2014. MEDLINE with full text, PsychINFO, CINAHL, ERIC and ProQuest were systematically searched 
[22,31-34]. The following search terms were used: (university OR college) AND (health promotion OR intervention OR program OR education) AND (behavior OR physical activity OR exercise OR diet OR nutrition OR weight). Published articles in peer reviewed journals were considered for the review. Bibliographies of selected studies were also considered. Only manuscripts written in English were considered for the review. Two reviewers independently assessed articles for study inclusion, initially based on the title and abstract. Full texts were then retrieved and assessed for inclusion. A third reviewer was used to make the final decision in the case of discrepancies.

\section{Study inclusion and exclusion criteria Type of participants}

Any study including students attending institutions within the tertiary education sector was included. If other types of participants, e.g., staff were also recruited, only students' data were extracted.

\section{Type of intervention (s)/phenomena of interest}

Interventions deemed eligible for inclusion had to be implemented within a tertiary education setting and have the aim of improving physical activity and/or dietary intake and/or weight. Interventions of all lengths were accepted for inclusion within the review.

\section{Type of studies}

All quantitative study designs (including randomized controlled trials, non-randomized experimental trials, prepost with no control group) were eligible for inclusion.

\section{Type of outcomes}

This review considered the following outcome measures specific to the health behavior targeted (an increase in knowledge among participants was not a sufficient outcome):

i) Physical activity related outcomes: steps per day, time spent undertaking vigorous and/or moderate physical activity, $\mathrm{VO}_{2}$ max, muscular strength/ endurance, energy expenditure, flexibility;

ii) Nutrition outcomes: energy intake, macronutrient composition, core food group consumption, diet quality; and,

iii) Weight related outcomes: weight (kg or lbs), body mass index $\left(\mathrm{kg} / \mathrm{m}^{2}\right)$ (BMI), waist circumference (cm), \% weight loss, \% body fat, waist-to-hip ratio (WHR).

\section{Risk of bias}

Risk of bias was assessed for all included studies by two independent reviewers (SK, SR) (a third reviewer [SC] was consulted and consensus reached in the event of a disagreement) using the Academy of Nutrition and
Dietetics Quality Criteria Checklist: Primary Research tool assessing 10 criteria [35]. These criteria included whether: (1) The study clearly stated the research question; (2) If the selection of participants was free from bias; (3) If the study groups were comparable; (4) Description of method of handling withdrawals; (5) Use of blinding; (6) Detailed description of interventions and comparisons; (7) Clear definition of outcomes and valid and reliable measurements; (8) Appropriate statistical analysis; (9) Consideration of limitations; and, (10) Likelihood of bias due to funding. Study quality was classed as positive if criteria 2, 3, 6 and 7, as well as one other validity criteria question were scored with a 'yes', neutral if criteria points 2, 36 and 7 did not score a 'yes', or negative if more than six of the validity criteria questions were answered with a 'no'.

\section{Data extraction}

Data extraction was performed by two reviewers (SK, SR) using a standardized form developed by the researchers. The data extraction consisted of 11 dimensions; country of origin, target sample and size, participants' mean age, duration of study, intervention description, participant retention, health behavior, study design, outcomes, results, and significance of results (see Additional file 1: Table S1, Additional file 2: Table S2, and Table 1). Extraction was checked for accuracy and consistency by a third reviewer (SC).

\section{Meta-analysis}

Results were pooled in meta-analysis if they were available as final values at post-intervention, the number of participants was recorded and interventions were sufficiently similar for comparison. If standard deviations were not available, but other statistics (e.g., 95\% CI or standard errors) were available, they were converted according to the calculations outlined in the Cochrane Handbook for Systematic Reviews of Interventions [36]. Heterogeneity was assessed using chi-squared with significant heterogeneity assigned at a $P$ value $<0.10$. If significant heterogeneity existed, the random effects model was used for statistical analysis; if homogenous, the fixed effect model was used. The data from individual studies on physical activity were combined across studies using standardized mean difference (SMD) due to the differences in reported metrics for total, moderate and vigorous physical activity. A unit conversion was not undertaken. Therefore, the meta-analytical results do not reflect the specific magnitude of effects for each study, but rather the extent to which they are more successful against controls. When a study compared multiple treatment groups with a single control, the sample size of the control was divided equally across the treatment group arms so the participants were not counted more than once in the analysis. All meta-analyses were conducted using Review Manager 
Table 1 Critical appraisal criteria of study methodologies

\begin{tabular}{|c|c|c|c|c|c|c|c|c|c|c|c|}
\hline Study & $\begin{array}{l}\text { Criteria } \\
1\end{array}$ & $\begin{array}{l}\text { Criteria } \\
2\end{array}$ & $\begin{array}{l}\text { Criteria } \\
3\end{array}$ & $\begin{array}{l}\text { Criteria } \\
\mathbf{4}\end{array}$ & $\begin{array}{l}\text { Criteria } \\
5\end{array}$ & $\begin{array}{l}\text { Criteria } \\
6 \\
\end{array}$ & $\begin{array}{l}\text { Criteria } \\
7\end{array}$ & $\begin{array}{l}\text { Criteria } \\
\mathbf{8} \\
\end{array}$ & $\begin{array}{l}\text { Criteria } \\
9\end{array}$ & $\begin{array}{l}\text { Criteria } \\
10\end{array}$ & Classification \\
\hline 1. Abu-Moghli et al. 2010 [1] & 1 & 0 & 0 & 0 & 0 & 0 & 1 & 1 & 1 & 1 & $\varnothing$ \\
\hline 2. Afifi Soweid et al. 2003 [69] & 1 & 0 & 0 & 0 & 0 & 1 & 0 & 0 & 1 & 1 & - \\
\hline 3. Alpar et al. 2008 [67] & 1 & 0 & 0 & 1 & 0 & 1 & 1 & 1 & 0 & 1 & $\varnothing$ \\
\hline 4. Bowden et al. 2007 [37] & 1 & 1 & 0 & 0 & 0 & 1 & 0 & 1 & 1 & 1 & $\varnothing$ \\
\hline 5. Boyle et al. 2011 [38] & 1 & 0 & 1 & 0 & 0 & 1 & 0 & 1 & 1 & 1 & $\varnothing$ \\
\hline 6. Brown et al. 2011 [39] & 1 & 0 & 0 & 0 & 0 & 1 & 1 & 1 & 1 & 1 & $\varnothing$ \\
\hline 7. Buscemi et al. 2011 [40] & 1 & 1 & 1 & 0 & 0 & 1 & 0 & 1 & 1 & 1 & $\varnothing$ \\
\hline 8. Cardinal et al. 2002 [41] & 1 & 0 & 0 & 0 & 0 & 1 & 1 & 1 & 1 & 1 & $\varnothing$ \\
\hline 9. Cavallo et al. 2012 [19] & 1 & 0 & 1 & 0 & 0 & 1 & 1 & 1 & 1 & 1 & $\varnothing$ \\
\hline 10. Chen et al. 1989 [42] & 1 & 0 & 0 & 0 & 0 & 1 & 0 & 0 & 0 & 1 & - \\
\hline 11. Claxton et al. 2009 [43] & 1 & 0 & 0 & 1 & 0 & 0 & 1 & 1 & 0 & 1 & $\varnothing$ \\
\hline 12. Evans \& Mary 2002 [43] & 1 & 0 & 0 & 0 & 0 & 1 & 0 & 1 & 0 & 1 & - \\
\hline 13. Fischer \& Bryant 2008 [45] & 1 & 1 & 1 & 0 & 0 & 0 & 1 & 1 & 1 & 1 & $\varnothing$ \\
\hline 14. Gieck \& Olsen 2007 [46] & 1 & 1 & 0 & 0 & 0 & 1 & 1 & 1 & 1 & 1 & $\varnothing$ \\
\hline 15. Gow et al. 2010 [47] & 0 & 1 & 1 & 1 & 0 & 1 & 1 & 1 & 1 & 1 & + \\
\hline 16. Gray et al. 1987 [47] & 0 & 1 & 0 & 0 & 0 & 0 & 0 & 0 & 1 & 1 & - \\
\hline 17. Grim et al. 2011 [5] & 1 & 0 & 0 & 1 & 0 & 1 & 1 & 1 & 1 & 1 & $\varnothing$ \\
\hline 18. Ha \& Caine-Bish 2009 [49] & 1 & 0 & 0 & 0 & 0 & 1 & 1 & 1 & 1 & 1 & $\varnothing$ \\
\hline 19. Hager et al. 2012 [50] & 1 & 1 & 0 & 0 & 0 & 1 & 1 & 1 & 1 & 1 & $\varnothing$ \\
\hline 20. Harvey-Berino et al. 2012 [52] & 1 & 0 & 0 & 0 & 0 & 1 & 0 & 0 & 1 & 1 & - \\
\hline 21. Hekler et al. 2010 [51] & 1 & 0 & 0 & 0 & 0 & 1 & 1 & 1 & 1 & 1 & $\varnothing$ \\
\hline 22. Huang et al. 2009 [7] & 1 & 1 & 1 & 0 & 0 & 1 & 1 & 1 & 1 & 1 & + \\
\hline 23. Ince 2008 [68] & 1 & 1 & 0 & 0 & 0 & 1 & 0 & 1 & 0 & 1 & $\varnothing$ \\
\hline 24. Kolodinsky et al. 2008 [53] & 1 & 1 & 0 & 0 & 0 & 0 & 0 & 0 & 1 & 1 & - \\
\hline 25. Lachausse 2012 [54] & 1 & 1 & 0 & 0 & 0 & 1 & 1 & 1 & 1 & 1 & $\varnothing$ \\
\hline 26. LeCheminant et al. 2011 [55] & 1 & 1 & 0 & 0 & 0 & 1 & 1 & 1 & 1 & 1 & $\varnothing$ \\
\hline 27. Magoc et al. 2011 [57] & 1 & 1 & 1 & 0 & 0 & 1 & 1 & 1 & 1 & 1 & + \\
\hline 28. Martens et al. 2012 [40] & 1 & 1 & 1 & 0 & 1 & 1 & 0 & 1 & 1 & 1 & $\varnothing$ \\
\hline 29. McClary King et al. 2013 [56] & 1 & 0 & 0 & 0 & 0 & 1 & 1 & 1 & 1 & 1 & $\varnothing$ \\
\hline 30. Musgrave \& Thornbury 1976 [59] & 0 & 1 & 0 & 0 & 0 & 1 & 0 & 0 & 0 & 1 & - \\
\hline 31. Pearce \& Cross 2013 [31] & 1 & 0 & 0 & 0 & 0 & 1 & 1 & 1 & 1 & 1 & $\varnothing$ \\
\hline 32. Pearman et al. 1997 [60] & 0 & 1 & 0 & 0 & 0 & 0 & 0 & 1 & 1 & 1 & - \\
\hline 33. Peterson et al. 2010 [61] & 1 & 1 & 0 & 0 & 0 & 1 & 0 & 1 & 1 & 1 & $\varnothing$ \\
\hline 34. Reed et al. 2011 [62] & 1 & 1 & 0 & 0 & 1 & 1 & 0 & 1 & 1 & 1 & $\varnothing$ \\
\hline 35. Sallis et al. 1999 [63] & 1 & 1 & 0 & 0 & 0 & 1 & 1 & 1 & 1 & 1 & $\varnothing$ \\
\hline 36. Skar et al. 2011 [70] & 1 & 1 & 1 & 1 & 1 & 1 & 0 & 1 & 0 & 1 & $\varnothing$ \\
\hline 37. Tully \& Cupples 2011 [71] & 1 & 1 & 0 & 0 & 1 & 1 & 0 & 1 & 1 & 1 & $\varnothing$ \\
\hline 38. Wadsworth et al. [64] & 1 & 1 & 0 & 0 & 0 & 1 & 1 & 1 & 0 & 1 & $\varnothing$ \\
\hline 39. Werch et al. 2007 [65] & 1 & 1 & 1 & 1 & 0 & 1 & 1 & 1 & 1 & 1 & + \\
\hline
\end{tabular}


Table 1 Critical appraisal criteria of study methodologies (Continued)

\begin{tabular}{|c|c|c|c|c|c|c|c|c|c|c|c|}
\hline 40. Werch et al. 2008 [73] & 1 & 1 & 0 & 1 & 0 & 1 & 1 & 1 & 1 & 1 & $\varnothing$ \\
\hline \multirow[t]{2}{*}{ 41. Yakusheva et al. 2011 [66] } & 1 & 1 & 0 & 0 & 0 & 0 & 0 & 1 & 1 & 1 & $\varnothing$ \\
\hline & 37 & 25 & 10 & 7 & 4 & 34 & 23 & 35 & 33 & 41 & \\
\hline
\end{tabular}

Criteria: 1) Was the research question clearly stated? 2) Was the selection of study subjects/patients free from bias? 3) Were study groups comparable? 4) Was the method of handling withdrawals described? 5) Was blinding used to prevent introduction of bias? 6) Were intervention/therapeutic regimens/exposure factor or procedure and any comparison(s) described in detail? Were intervening factors described? 7) Were outcomes clearly defined and the measurements valid and reliable? 8) Was the statistical analysis appropriate for the study design and type of outcome indicators? 9) Were conclusions supported by results with biases and

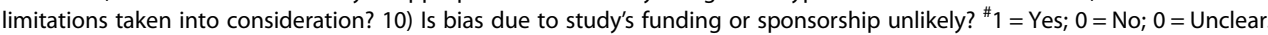

MINUS/NEGATIVE (-) If most (six or more) of the answers to the above validity questions are "No", the report should be designated with a minus (-) symbol on the Evidence Worksheet.

NEUTRAL $(\varnothing)$ If the answers to validity criteria questions $2,3,6$, and 7 do not indicate that the study is exceptionally strong, the report should be designated with a neutral $(\varnothing)$ symbol on the Evidence Worksheet.

PLUS/POSITIVE (+) If most of the answers to the above validity questions are "Yes" (including criteria 2, 3, 6, 7 and at least one additional "Yes"), the report should be designated with a plus symbol (+) on the Evidence Worksheet.

5.2; the results were calculated by weighting the amount of information they contribute i.e., the inverse variances of their effect estimates. Intention-to-treat analyses were used where available/reported in the studies. If this information was not available completers analyses was used.

\section{Results}

Overall, 41 studies targeting improvements in student health outcomes (physical activity, diet, weight loss) within tertiary education settings met the inclusion/exclusion criteria. Study characteristics (i.e., country, target sample and size, age, duration, intervention and retention) and risk of bias scores are summarised in Additional file 1: Table S1. Risk of bias assessment indicated eight studies had a negative rating (high risk of bias), 30 were considered neutral, and the remaining four had a positive rating (low risk of bias).

Of the 41 studies identified, 33 were conducted in the United States [5,19,37-66], two in Turkey [67,68] and one each in Jordan [1], Lebanon [69], Scotland [70], Ireland [71], Taiwan [72] and Australia [9]. Study designs included randomized controlled trials $(n=16)$, nonrandomized controlled trials $(n=12)$ and pre-post designs with no control group $(\mathrm{n}=13)$. Study durations ranged from a 30-minute one-on-one brief motivational intervention [58] to an intervention spanning four academic calendar years [67]. Weight loss was the sole focus in two studies [52,59], physical activity in 11 studies $[5,19,41,43,45,46,57,58,63,70,72]$, and nutrition was the focus of 10 studies [9,39,42,44,48,49,51,53,61,62]. A combination of weight loss and/or physical activity and/or nutrition outcomes were examined by 18 studies [1,37,38,40,47,50,54-56,60,64-69,71,73]. Study participant numbers ranged from 16 [53] to 2971 [50]. Retention rates ranged from $36.6 \%$ [46] to $100 \%$ [49,51,53,62,71]; five studies $[19,42,44,66,68]$ did not report retention rates.

Three studies did not report the sex distribution of participants $[41,57,68]$. Of the remaining studies, four had even sex distribution $[48,56,62,69]$, three had a majority of male participants [43,55,61]; 23 studies were comprised of majority female participants [1,5,9,37-40,42,44,47,49,50,52-54,58-60,63,65,70,71,73]; and eight studies consisted of entirely female samples $[19,45,46,51,64,66,67,72]$. In general, demographic characteristics of participants such as age were not consistently reported.

\section{Effectiveness of interventions \\ Physical activity and fitness outcomes}

As represented in Additional file 2: Table S2, of the 41 studies in our review, 29 examined physical activity (11 exclusively, 18 in combination with other health behaviors). Of these 29 studies, the average risk of bias classification was neutral. Of the studies investigating change in physical activity or fitness behavior, 18 of 29 reported significant improvements from pre- to post-intervention. In five studies a significant increase in physical activity minutes was achieved [50,57,63,70,71]; in five studies the number of days participating in physical activity increased $[5,43,58,64,65]$; exercise duration increased in three studies [38,65,67]; Metabolic Equivalent of Task (METs) increased in three studies [60,68,72]; exercise barriers decreased in one study [56]; and Physical Activity Readiness Questionnaire (PAR-Q) scores improved in one study [50].

\section{Meta-analysis \\ Total physical activity}

Five studies comparing interventions targeting health behaviors to a control condition that assessed total physical activity level at post-intervention were combined in meta-analysis (see Figure 1). Three of the studies included multiple intervention arms compared to a single control group, therefore 10, intervention versus control comparisons were included in the analysis.

The studies were significantly heterogeneous $\left(\chi^{2}=25.65\right.$, d.f. $\left.=9[P=0.002], I^{2}=65 \%\right)$ and demonstrated no significant difference in total physical activity between intervention and control groups at post-intervention (SMD -0.11 $[-0.30,0.08], \mathrm{Z}=1.13 P=0.26)$. 


\begin{tabular}{|c|c|c|c|c|c|c|c|c|c|c|}
\hline \multicolumn{11}{|c|}{ Panel 1: Total Physical Activity } \\
\hline \multirow[b]{2}{*}{ Study or Subgroup } & \multicolumn{4}{|c|}{ Intervention } & \multicolumn{2}{|c|}{ Control } & \multicolumn{3}{|r|}{ Std. Mean Difference } & \multirow{2}{*}{$\begin{array}{l}\text { Std. Mean Difference } \\
\text { IV, Random, } 95 \% \mathrm{Cl}\end{array}$} \\
\hline & & Mean & $\mathrm{SD}$ & Total & Mean & SD & Total $\mathrm{V}$ & Weight & IV, Random, $95 \% \mathrm{Cl}$ & \\
\hline \multicolumn{2}{|l|}{ Gow 2010 Combined } & 4,119 & 3,561 & 40 & 4,304 & 3,542 & 13 & $6.1 \%$ & $-0.05[-0.68,0.57]$ & \\
\hline Gow 2010 Feedback & & 5,005 & 3,597 & 40 & 4,304 & 3,542 & 13 & $6.1 \%$ & $0.19[-0.43,0.82]$ & \\
\hline Gow 2010 Internet & & 3,059 & 3,379 & 39 & 4,304 & 3,542 & 13 & $6.0 \%$ & $-0.36[-0.99,0.27]$ & - \\
\hline \multicolumn{2}{|l|}{ Huang 2009 Stage-Matched } & $34.84 \quad 5$ & 575.87 & 451 & $1,172.33$ & 661.62 & 22 & $7.5 \%$ & $-0.88[-1.41,-0.35]$ & - \\
\hline \multicolumn{2}{|l|}{ Huang 2010 Generic } & 40.068 & 826.83 & 421 & $1,172.33$ & 661.62 & 22 & $7.4 \%$ & $-0.81[-1.34,-0.27]$ & - \\
\hline \multicolumn{2}{|l|}{ Skar 2011 AP } & 2.96 & 2.26 & 310 & 2.96 & 2.31 & 105 & $14.4 \%$ & $0.00[-0.22,0.22]$ & \\
\hline \multirow{2}{*}{\multicolumn{2}{|c|}{ Skar $2011 \mathrm{AP}+\mathrm{CP}$}} & 2.98 & 2.3 & 225 & 2.96 & 2.31 & 105 & $14.2 \%$ & $0.01[-0.22,0.24]$ & \\
\hline & Skar $2011 \mathrm{CP}$ & 2.76 & 2.3 & 335 & 2.96 & 2.31 & 105 & $14.5 \%$ & $-0.09[-0.31,0.13]$ & \\
\hline \multicolumn{2}{|l|}{ Wadsworth 2010} & 3.06 & 2.22 & 45 & 2.03 & 1.83 & 46 & $9.6 \%$ & $0.50[0.08,0.92]$ & - \\
\hline \multicolumn{2}{|l|}{ Werch 2008} & 3.66 & 1.54 & 140 & 3.75 & 1.56 & 143 & $14.1 \%$ & $-0.06[-0.29,0.18]$ & \\
\hline \multicolumn{2}{|l|}{ Total (95\% Cl) } & & & 1261 & & & $587 \quad 1$ & $100.0 \%$ & $-0.11[-0.30,0.08]$ & \\
\hline \multicolumn{7}{|c|}{$\begin{array}{l}\text { Heterogeneity: } \text { Tau }^{2}=0.05 ; C h i^{2}=25.65, d i=9(P=0.002) ; I^{2}=65 \% \\
\text { Test for overall effect: } Z=1.13(P=0.26)\end{array}$} & & & & \begin{tabular}{ll|lll} 
& 1 & 1 & 1 \\
-10 & -5 & 0 & 5 & 10 \\
& Favours control Favours intervention
\end{tabular} \\
\hline \multicolumn{11}{|c|}{ Panel 2: Vigorous Physical Activity } \\
\hline \multirow[b]{2}{*}{ Study or Subgroup } & \multicolumn{3}{|c|}{ Intervention } & \multicolumn{2}{|r|}{ Control } & & & \multicolumn{2}{|c|}{ Std. Mean Difference } & Std. Mean Difference \\
\hline & Mean & SD & Total & I Mean & SD & Total & Weight & IV, R & Random, $95 \% \mathrm{Cl}$ & IV, Random, $95 \% \mathrm{Cl}$ \\
\hline Buscemi 2011 & 68.97 & 97.71 & 34 & 74.87 & 194.5 & 36 & $12.5 \%$ & & $0.04[-0.51,0.43]$ & $T$ \\
\hline Claxton 2009 & 2.19 & 1.73 & 3164 & $+\quad 2.19$ & $9 \quad 1.82$ & 201 & $15.2 \%$ & & $0.00[-0.21,0.21]$ & \\
\hline Gow 2010 Combined & 939 & 1,448 & 40 & 1,270 & 1,429 & 13 & $10.6 \%$ & & $0.23[-0.85,0.40]$ & - \\
\hline Gow 2010 Feedback & 1,479 & 1,448 & 40 & 1,270 & 1,429 & 13 & $10.6 \%$ & & $0.14[-0.48,0.77]$ & - \\
\hline Gow 2010 Internet & 909 & 1,393 & 39 & 1,270 & 1,429 & 13 & $10.6 \%$ & & $0.25[-0.88,0.38]$ & \\
\hline Grim 2007 PA group & 2.36 & 1.84 & 64 & $+\quad 0.39$ & $9 \quad 0.61$ & 30 & $12.5 \%$ & & $1.25[0.78,1.72]$ & $=$ \\
\hline Grim $2007 \mathrm{Web}$ & 2.21 & 1.84 & $4 \quad 108$ & 0.39 & 90.61 & 30 & $13.0 \%$ & & $1.09[0.67,1.52]$ & - \\
\hline Werch 2008 & 4.19 & 1.67 & 143 & 3.97 & $7 \quad 1.66$ & 140 & $15.0 \%$ & & $0.13[-0.10,0.37]$ & \\
\hline Total $(95 \% \mathrm{Cl})$ & & & 632 & & & 476 & $100.0 \%$ & & $.28[-0.08,0.63]$ & \\
\hline $\begin{array}{l}\text { Heterogeneity: Tau }{ }^{2}=0 \\
\text { Test for overall effect: } Z\end{array}$ & $\begin{array}{l}20 ; \mathrm{Ch}^{3} \\
=1.54(\mathrm{I}\end{array}$ & $\begin{array}{l}\mathrm{j}^{\mathrm{z}}=44.8 \\
(P=0.12\end{array}$ & $\begin{array}{l}\text { 86, } d f=7 \\
\text { 2) }\end{array}$ & $7(P<0$. & $0.00001) ;$ & $I^{2}=849$ & & & -10 & 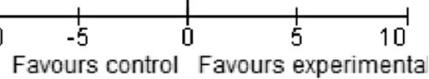 \\
\hline Panel 3: Moderate & Physic & cal Act & tivity & & & & & & & \\
\hline & & terventio & & & Control & & & Std. Me & ean Difference & Std. Mean Difference \\
\hline Study or Subgroup & Mean & SD & Total & Mean & $\mathrm{SD}$ & Total & Weight & & V, Fixed, $95 \% \mathrm{Cl}$ & IV, Fixed, $95 \% \mathrm{Cl}$ \\
\hline Buscemi 2011 & 69.62 & 79.11 & 34 & 66 & 93.67 & 36 & $7.0 \%$ & & $0.04[-0.43,0.51]$ & $t$ \\
\hline Claxton 2009 & 3.11 & $1.6 ?$ & 164 & 2.82 & 1.6 & 201 & $36.0 \%$ & & $0.18[-0.03,0.38]$ & 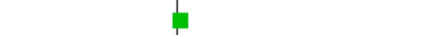 \\
\hline Gow 2010 Combined & 914 & 1,464 & 40 & 772 & 21,455 & 13 & $3.9 \%$ & & $0.10[-0.53,0.72]$ & - \\
\hline Gow 2010 Feedback & 1,128 & 1,512 & 40 & 772 & 21,455 & 13 & $3.9 \%$ & & $0.23[-0.39,0.86]$ & - \\
\hline Gow 2010 Internet & 899.3 & 1,418 & 39 & 772 & 21,455 & 13 & $3.9 \%$ & & $0.09[-0.54,0.72]$ & - \\
\hline Grim 2007 PA group & 3.53 & 2.03 & 64 & 2.28 & $3 \quad 2.19$ & 30 & $7.8 \%$ & & $0.60[0.15,1.04]$ & - \\
\hline Grim 2007 Web & 3.84 & 3.89 & 108 & 2.28 & 2.19 & 30 & $9.2 \%$ & & $0.43[0.02,0.84]$ & $=$ \\
\hline Werch 2008 & 4.52 & 1.78 & 140 & 4.46 & 1.67 & 143 & $28.3 \%$ & & $.03[-0.20,0.27]$ & 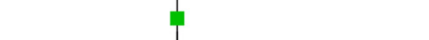 \\
\hline Total $(95 \% \mathrm{Cl})$ & & & 629 & & & 479 & $100.0 \%$ & & $0.18[0.06,0.30]$ & 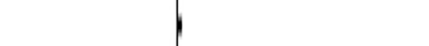 \\
\hline $\begin{array}{l}\text { Heterogeneity: } \text { Chi }^{2}=6 \text {. } \\
\text { Test for overall effect: } Z\end{array}$ & $\begin{array}{l}86, \mathrm{df}= \\
=2.84(\mathrm{~F}\end{array}$ & $\begin{array}{l}7(P=0 \\
(P=0.00\end{array}$ & $\begin{array}{l}0.44) ; F^{2}= \\
05)\end{array}$ & $=0 \%$ & & & & & $\stackrel{-10}{F}$ & 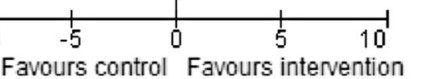 \\
\hline
\end{tabular}

Figure 1 Meta-analysis of total (panel 1), vigorous (panel 2) and moderate (panel 3) physical activity.

\section{Vigorous physical activity}

Five studies comparing interventions targeting health behaviors to a control condition that assessed vigorous physical activity levels at post-intervention were combined in a meta-analysis (see Figure 1). Two of the studies included multiple intervention arms compared to a single control group; therefore eight, intervention versus control comparisons were included in the analysis.

The studies were significantly heterogeneous $\left(\chi^{2}=\right.$ 44.86, d.f. $\left.=7[P<0.001], I^{2}=84 \%\right)$ and demonstrated no 
significant difference in vigorous physical activity levels between the intervention and control groups at postintervention (SMD $0.28[-0.08,0.63], \mathrm{Z}=1.54 \quad P=0.12$ ) (see Figure 1).

\section{Moderate physical activity}

As represented in Figure 1, five studies comparing interventions targeting health behaviors to a control condition that assessed moderate physical activity levels at post-intervention were combined in meta-analysis. Two of the studies included multiple intervention arms compared to a single control group; therefore eight, intervention versus control comparisons were included in the analysis.

The studies were homogenous $\left(\chi^{2}=6.86\right.$, d.f. $=7[P=$ $0.44], I^{2}=0 \%$ ) and demonstrated significantly greater moderate physical activity levels in the intervention group compared to the control group at post intervention (SMD 0.18 $[0.06,0.30], \mathrm{Z}=2.84 P=0.005)$.

\section{Nutrition outcomes}

Of the 41 included studies, 24 reported nutrition outcomes (10 examined nutrition exclusively), with fruit and vegetable intake the most reported outcome, used in 12 of the reported studies [39,40,44,47,49-51,54,56,62,65,73]. Of the 24 studies, the average risk of bias rating was neutral. Six studies had a negative rating, sixteen were neutral and two had a positive rating.

Interventions were found to be effective in improving nutrition behaviors in 12 of the 24 studies $[1,39,44,49-51,54$, 60-62,65,68]. In three studies a significant improvement in diet quality was achieved $[1,61,68]$; six studies reported vegetable intake increases [39,44,49-51,54]; in six studies fruit intake increased $[39,44,49,50,54,62]$; fat intake was reduced in four studies $[44,51,60,61]$; fewer calories were consumed in one study [60]; frequency of wholegrain product consumption was increased in one study [50]; and consumption of healthy fats increased in one study [65]. Due to the heterogeneity and lack of standard methods to assess dietary intake within the nutrition domain, a metaanalysis was unable to be conducted.

\section{Weight outcomes}

Of the 41 included studies, 12 reported weight-related outcomes (two examined weight exclusively) $[37,38,40,47,50$, $52,54,55,59,64,66,71]$ and of these 12 , four reported significant improvements in these outcomes $[38,47,52,66]$. The average risk of bias rating for the 12 studies was neutral. A significant reduction in waist-to-hip ratio was reported in one study [38]; in one study BMI decreased significantly [47]; one study reported significant weight loss [52]; and a significant increase in the number of participants trying to lose weight was reported by one study [66]. Due to the variation in aims and measures within the domain of weight, a meta-analysis was not conducted.

\section{Discussion}

The current review identified 41 studies that investigated the impact of lifestyle interventions targeting improvement of health outcomes (specifically physical activity, diet or weight) for students within the tertiary sector. Most studies reported at least one significant improvement in a health outcome variable, with a number of studies having multiple significant impacts. Study results were mostly positive, with at least half of the studies for physical activity and nutrition reporting significant outcomes. These included 18/29 studies examining physical activity that found significant effects including increased physical activity minutes, an increase in the number of days participating in physical activity and also in exercise duration, increased METs and PAR-Q scores and a decrease in barriers to exercise. In addition, results of the meta-analysis suggest the studies targeting moderate physical activity demonstrated significantly greater moderate physical activity levels in the intervention group compared to the control group at post intervention. Of the studies examining nutrition, $50 \%$ reported significant improvements, including improved diet quality, increased fruit, vegetable and wholegrain intake, and healthy fats and a reduction in overall fat intake and calories. Of the studies examining body weight, four of 12 resulted in significant outcomes including reductions in weight, BMI, and WHR and/or an increase in the number of participants trying to lose weight.

Interventions spanning a university semester or less ( $\leq 12$ weeks) generally resulted in a greater number of significant outcomes in comparison to interventions with a duration of more than a semester. In addition, interventions targeting nutrition only resulted in more significant outcomes in comparison to targeting PA, weight or multiple behaviours. For instance, of the ten interventions targeting dietary behaviour, eight $(80 \%)$ had significant results and the majority of these studies $(7 / 8)$ were $\leq 12$ weeks. For PA only studies, seven of the $11(64 \%)$ interventions had significant results, and of these seven studies five had a duration of $\leq 12$ weeks. Of the two studies examining weight only interventions, only the 12 week study reported significant results. Furthermore, when targeting a combination of behaviours, 11 of 18 (61\%) interventions had significant results, and just over half of these interventions had a duration of $\leq 12$ weeks $(6 / 11)$.

Less than half of the studies in each category were randomized controlled trials, however there was no trend (based on study counts) towards study design influencing the effectiveness of interventions. The vast majority of the studies were conducted in the USA $(n=31)$ and 
therefore the global generalizability of these results must be interpreted with caution.

With few exceptions, participant numbers were surprisingly small given the large institutions from which participants were drawn. Additionally, participants were overwhelmingly female, which may be due in part to the higher percentage of females enrolled in some universities and colleges. This raises questions about the approaches used to recruit participants or the intrinsic appeal of the interventions trialed. Indeed, results from a questionnaire examining gender differences in the health habits of university students showed that males were less interested in nutrition advice and health-enhancing behaviors, suggesting that interventions targeting health behaviors in university/ college students may need to be gender-specific to address the different needs and interests of both sexes [15].

The transition from secondary to tertiary education often results in an increase in health risk secondary to a decrease in physical activity and increase in poor dietary choices [74]. For many students, making the transition to tertiary education coincides with more freedom and control over their lives. However, this can contribute to the increases in risk taking behaviors that are evident in this population [75]. With this new-found independence, many students may not have developed skills such as selfefficacy and accountability, leaving them at higher risk of adopting unhealthy behaviors. A number of studies in this review successfully targeted self-efficacy $[5,7,39,46,54,57,64]$ to improve health behaviors.

Interventions that were embedded within university/ college courses were effective at improving physical activity, nutrition and weight-related outcomes. Courseembedded interventions involve frequent face-to-face contact with facilitators. It has been suggested that frequent professional contact may improve health outcomes by enhancing vigilance and providing encouragement and support [76]. Additionally, interventions where students received feedback on their progress appeared to be more effective than simply attending lectures or receiving educational resources.

Universities and colleges are an ideal setting for implementation of health promotion programs as they support a large student population at key time for the development of lifestyle skills and behaviors. Students have access to world-class facilities, technology, and highly educated staff including a variety of health disciplines, all of which could contribute to the development of highly effective health promoting interventions. A number of studies in this review utilized university facilities, such as fitness centres and designated walking tracks, showing significant improvements in physical activity outcomes. Besides ease of access for students, use of existing facilities and resources is also cost-effective, which is often a major limitation of health promotion programs.

\section{Conclusions}

This study extends the current literature examining the effectiveness of interventions targeting physical activity, nutrition and weight-loss behaviors amongst university and college students. To the best of the authors' knowledge it is the first systematic review examining health behaviors of students within a tertiary education setting. Some limitations of the field exist which should be acknowledged. First, the majority of studies examined were conducted in the USA, which may limit interpretations and global generalizability of results. Second, only four of the 41 studies that met the inclusion criteria showed a positive result in meeting the risk of bias validity criteria questions. Also, the potential effect of publication bias must be considered, as the observations made in this review did not include grey literature (e.g., unpublished dissertations).

This review has several strengths. It employed a comprehensive search strategy, adhered to the PRISMA protocol with two reviewers used for the identification and evaluation of studies [30], assessed study risk of bias with two independent reviewers using the Academy of Nutrition and Dietetics Quality Criteria Checklist, and included a meta-analyses for physical activity.

Tertiary education students within the university/college setting are ideal targets for lifestyle interventions aimed at improving health behaviors. Within this setting, students are often surrounded by an abundance of research expertise, multi-disciplinary health professionals, and worldclass facilities and resources making this potentially an ideal health-promoting environment. Additionally, students are in a learning environment and are still at an age where health behaviors that impact on health later in life can be improved. Therefore, there is significant scope for implementation of lifestyle interventions to improve the health of this group that represents a significant proportion of our population.

\section{Additional files}

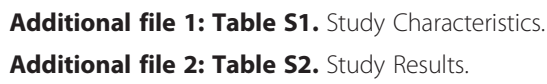

\section{Competing interests}

The authors declare that they have no competing interests.

Authors' contributions

RCP, SAC, CEC, RC and JG conceptualized and designed the study. SAC and JA drafted the introduction section. SAC, RLW, SGK, SLR extracted the data. $\mathrm{MJH}$ conducted the statistical analyses. All authors contributed to the writing and editing of the manuscript. All authors read and approved the final manuscript.

\section{Acknowledgements}

RCP is supported by a National Health \& Medical Research Council (NH \& MRC) Senior Research Fellowship. 


\section{Author details}

${ }^{1}$ Priority Research Centre for Physical Activity and Nutrition, University of Newcastle, Callaghan Campus, Newcastle, NSW, Australia. ${ }^{2}$ School of Education, Faculty of Education and Arts, University of Newcastle, Callaghan Campus, Newcastle, NSW, Australia. ${ }^{3}$ School of Health Sciences, Faculty of Health, University of Newcastle, Callaghan Campus, Newcastle, NSW, Australia. ${ }^{4}$ School of Biomedical Sciences and Pharmacy, Faculty of Health, University of Newcastle, Callaghan Campus, Newcastle, NSW, Australia. ${ }^{5}$ School of Humanities and Social Science, Faculty of Education and Arts, University of Newcastle, Callaghan Campus, Newcastle, NSW, Australia.

\section{Received: 11 August 2014 Accepted: 10 March 2015}

\section{Published online: 01 April 2015}

\section{References}

1. Abu-Moghli FA, Khalaf IA, Barghoti FF, Abu-Moghli FA, Khalaf IA, Barghot FF. The influence of a health education programme on healthy lifestyles and practices among university students. Int J Nurs Pract. 2010;16:35-42.

2. Australian Institute of Health and Welfare. Risk factors contributing to chronic disease. In: Book Risk factors contributing to chronic disease. Canberra: AlHW; 2012.

3. Reiner M, Niermann C, Jekauc D, Woll A. Long-term health benefits of physical activity-a systematic review of longitudinal studies. BMC Public Health. 2013;13:813.

4. Bonevski B, Guillaumier A, Paul C, Walsh R. The vocational education setting for health promotion: a survey of students' health risk behaviours and preferences for help. Health Promot J Austr. 2014;24:185-91.

5. Grim M, Hortz B, Petosa R, Grim M, Hortz B, Petosa R. Impact evaluation of a pilot web-based intervention to increase physical activity. Am J Health Promot. 2011;25:227-30.

6. Haase A, Steptoe A, Sallis JF, Wardle J. Leisure-time physical activity in university students from 23 countries: associations with health beliefs, risk awareness, and national economic development. Prev Med. 2004;39:182-90.

7. Huang TT-K, Harris KJ, Lee RE, Nazir N, Born W, Kaur H. Assessing overweight, obesity, diet, and physical activity in college students. J Am Coll Health. 2003;52:83-6.

8. Irwin JD. Prevalence of university students' sufficient physical activity: a systematic review. Percept Mot Skills. 2004;98:927-43.

9. Weinstock J. A review of exercise as intervention for sedentary hazardous drinking college students: rationale and issues. J Am Coll Health. 2010:58:539-44.

10. Australian Bureau of Statistics. Australian Health Survey: First Results. In: Book Australian Health Survey: First Results. Canberra: ABS; 2012

11. Wirt A, Collins CE. Diet quality-what is it and does it matter? Public Health Nutr. 2009;12:2473-92.

12. National Health and Medical Research Council. Australian Dietary Guidelines. In: Book Australian Dietary Guidelines. Canberra: NHMRC; 2013.

13. Davy SR, Benes BA, Driskell JA. Sex differences in dieting trends, eating habits, and nutrition beliefs of a group of midwestern college students. J Am Diet Assoc. 2006;106:1673-7.

14. Haberman S, Luffey D. Weighing in college students' diet and exercise behaviors. J Am Coll Health. 1998:46:189-91.

15. Von Bothmer MI, Fridlund B. Gender differences in health habits and in motivation for a healthy lifestyle among Swedish university students. Nurs Health Sci. 2005;7:107-18.

16. McKinney CE. Assessment of dietary behaviors of college students participating in the health promotion program BUCS: live well. 2013.

17. El Ansari W, Stock C, John J, Deeny P, Phillips C, Snelgrove S, et al. Health promoting behaviours and lifestyle characteristics of students at seven universities in the UK. Cent Eur J Public Health. 2011;19:197.

18. Kattelmann KK, White AA, Greene GW, Byrd-Bredbenner C, Hoerr SL, Horacek TM, et al. Development of Young Adults Eating and Active for Health (YEAH) internet-based intervention via a community-based participatory research model. J Nutr Educ Behav. 2014;46:S10-25.

19. Cavallo DN, Tate DF, Ries AV, Brown JD, DeVellis RF, Ammerman AS. A social media-based physical activity intervention: a randomized controlled trial. Am J Prev Med. 2012;43:527-32.

20. Gropper SS, Simmons KP, Connell L, Ulrich PV. Changes in body weight, composition, and shape: a 4-year study of college students. Appl Physiol Nutr Metab. 2012;37:1118-23.
21. Fedewa MV, Das BM, Evans EM, Dishman RK. Change in weight and adiposity in college students: a systematic review and meta-analysis. Am J Prev Med. 2014;47:641-52.

22. Small M, Bailey-Davis L, Morgan N, Maggs J. Changes in eating and physical activity behaviors across seven semesters of college living on or off campus matters. Health Educ Behav. 2012;134:885-91.

23. Buckworth J, Nigg C. Physical activity, exercise, and sedentary behavior in college students. J Am Coll Health. 2004;53:28-34.

24. Nelson MC, Kocos R, Lytle LA, Perry CL. Understanding the perceived determinants of weight-related behaviors in late adolescence: a qualitative analysis among college youth. J Nutr Educ Behav. 2009;41:287-92.

25. United Nations Educational Scientific and Cultural Organization. Trends in Global Higher Education: Tracking an Academic Revolution. In: Book Trends in Global Higher Education: Tracking an Academic Revolution. Paris: UNESCO; 2009.

26. Davis DV, Mackintosh B. Making a Difference: Australian International Education. Sydney: New South Publishing; 2012.

27. Fredman P. Universities as role models for sustainable development. In European University Association Annual Conference: The Sustainability of European Universities. Warwick; 2012.

28. Knight A, La Placa V. Healthy universities: taking the university of Greenwich healthy universities initiative forward. Int J Health Promot Educ. 2013;51:41-9.

29. Zins JE. Building academic success on social and emotional learning: What does the research say?: Teachers College Press. 2004.

30. Liberati A, Altman DG, Tetzlaff J, Mulrow C, Gøtzsche PC, loannidis JPA, et al. The PRISMA statement for reporting systematic reviews and meta-analyses of studies that evaluate health care interventions: explanation and elaboration. PLoS Med. 2009:6:e1000100.

31. Livhits M, Mercado C, Yermilov I, Parikh JA, Dutson E, Mehran A, et al. Is social support associated with greater weight loss after bariatric surgery?: a systematic review. Obes Rev. 2011;12:142-8.

32. Sanders L, Huang J, Krumholz HM, Olkin I, Gardner CD, Bravata DM. Efficacy and safety of Low-carbohydrate diets: a systematic review. J Am Med Assoc. 2003;289:1837-50.

33. Franz MJ, VanWormer JJ, Crain AL, Boucher JL, Histon T, Caplan W, et al. Weight-loss outcomes: a systematic review and meta-analysis of weight-loss clinical trials with a minimum 1-year follow-up. J Am Diet Assoc. 2007:107:1755-67.

34. Weinheimer EM, Sands LP, Campbell WW. A systematic review of the separate and combined effects of energy restriction and exercise on fat-free mass in middle-aged and older adults: implications for sarcopenic obesity. Nutr Rev. 2010:68:375-88

35. Evidence Analysis Manual: Steps in the Academy Evidence Analysis Process. [http://www.adaevidencelibrary.com/files/Docs/2012_Jan_EA_Manual.pdf]

36. Higgins JP, Green S. Cochrane handbook for systematic reviews of interventions: Version 4.2.5. The Cochrane Library: Wiley Blackwell; 2005.

37. Bowden RG, Lanning BA, Doyle El, Slonaker B, Johnston HM, Scanes G, et al. Systemic glucose level changes with a carbohydrate-restricted and higher protein diet combined with exercise. J Am Coll Health. 2007;56:147-52.

38. Boyle J, Mattern CO, Lassiter JW, Ritzler JA, Boyle J, Mattern CO, et al. Peer 2 peer: efficacy of a course-based peer education intervention to increase physical activity among college students. J Am Coll Health. 2011;59:519-29.

39. Brown KN, Wengreen HJ, Vitale TS, Anderson JB, Brown KN, Wengreen HJ, et al. Increased self-efficacy for vegetable preparation following an online, skill-based intervention and in-class tasting experience as a part of a general education college nutrition course. Am J Health Promot. 2011;26:14-20.

40. Buscemi J, Yurasek AM, Dennhardt AA, Martens MP, Murphy JG. A randomized trial of a brief intervention for obesity in college students. Clin Obes. 2011;1:131-40.

41. Cardinal BJ, Jacques KM, Levy SS. Evaluation of a university course aimed at promoting exercise behavior. J Sports Med Phys Fitness. 2002;42:113-9.

42. Chen Jr MS, Minton JP, Adams B. Changing college students' lifestyles in favor of cancer prevention: a case study. J Cancer Educ. 1989:4:49-54.

43. Claxton D, Wells GM, Claxton D, Wells GM. The effect of physical activity homework on physical activity among college students. J Phys Act Health. 2009;6:203-10.

44. Evans AE, Mary KS, Evans AE, Mary KS-M. The right bite program: a theory-based nutrition intervention at a minority college campus. J Am Diet Assoc. 2002;102:S89-93. 
45. Fischer DV, Bryant J, Fischer DV, Bryant J. Effect of certified personal trainer services on stage of exercise behavior and exercise mediators in female college students. J Am Coll Health. 2008;56:369-76.

46. Gieck DJ, Olsen S, Gieck DJ, Olsen S. Holistic wellness as a means to developing a lifestyle approach to health behavior among college students. J Am Coll Health. 2007;56:29-35.

47. Gow RW, Trace SE, Mazzeo SE, Gow RW, Trace SE, Mazzeo SE. Preventing weight gain in first year college students: an online intervention to prevent the "freshman fifteen". Eat. 2010;11:33-9.

48. Gray CH, Colome JS, Curry-Daly JR. Elective cancer education: how effective from the public health viewpoint? Am J Public Health. 1987;77:1207-9.

49. Ha EJ, Caine-Bish N, Ha E-J, Caine-Bish N. Effect of nutrition intervention using a general nutrition course for promoting fruit and vegetable consumption among college students. J Nutr Educ Behav. 2009:41:103-9.

50. Hager R, George JD, LeCheminant JD, Bailey BW, Vincent WJ, Hager R, et al. Evaluation of a university general education health and wellness course delivered by lecture or online. Am J Health Promot. 2012;26:263-9.

51. Hekler EB, Gardner CD, Robinson TN, Hekler EB, Gardner CD, Robinson TN. Effects of a college course about food and society on students' eating behaviors. Am J Prev Med. 2010;38:543-7.

52. Harvey-Berino J, Pope L, Gold BC, Leonard H, Belliveau C, Harvey-Berino J, et al. Undergrad and overweight: an online behavioral weight management program for college students. J Nutr Educ Behav. 2012;44:604-8.

53. Kolodinsky J, Green J, Michahelles M, Harvey-Berino JR, Kolodinsky J, Green J, et al. The use of nutritional labels by college students in a food-court setting. J Am Coll Health. 2008:57:297-302.

54. Lachausse RG, Lachausse RG. My student body: effects of an internet-based prevention program to decrease obesity among college students. J Am Coll Health. 2012:60:324-30

55. LeCheminant JD, Smith JD, Covington NK, Hardin-Renschen T, Heden T, LeCheminant JD, et al. Pedometer use in university freshmen: a randomized controlled pilot study. Am J Health Behav. 2011;35:777-84.

56. McClary King K, Ling J, Ridner SL, Jacks DE, Newton KS, Topp RV. Fit Into College II: physical activity and nutrition behavior effectiveness and programming recommendations. Recreation Sports J. 2013;37:29-41.

57. Magoc D, Tomaka J, Bridges-Arzaga A, Magoc D, Tomaka J, Bridges-Arzaga A. Using the web to increase physical activity in college students. Am J Health Behav. 2011;35:142-54.

58. Martens MP, Buscemi J, Smith AE, Murphy JG, Martens MP, Buscemi J, et al. The short-term efficacy of a brief motivational intervention designed to increase physical activity among college students. J Phys Act Health. 2012;9:525-32.

59. Musgrave KO, Thornbury ME. Weight control program for university students conducted by nutrition seniors. J Am Diet Assoc. 1976;68:462-6.

60. Pearman 3rd SN, Valois RF, Sargent RG, Saunders RP, Drane JW, Macera CA. The impact of a required college health and physical education course on the health status of alumni. J Am Coll Health. 1997:46:77-85.

61. Peterson S, Duncan DP, Null DB, Roth SL, Gill L, Peterson S, et al. Positive changes in perceptions and selections of healthful foods by college students after a short-term point-of-selection intervention at a dining hall. J Am Coll Health. 2010;58:425-31.

62. Reed JA, Powers A, Greenwood M, Smith W, Underwood R, Reed JA, et al. Using "point of decision" messages to intervene on college students' eating behaviors. Am J Health Promot. 2011;25:298-300.

63. Sallis JF, Calfas KJ, Nichols JF, Sarkin JA, Johnson MF, Caparosa S, et al. Evaluation of a university course to promote physical activity: project GRAD. Res Q Exerc Sport. 1999;70:1-10.

64. Wadsworth DD, Hallam JS, Wadsworth DD, Hallam JS. Effect of a web site intervention on physical activity of college females. Am J Health Behav. 2010:34:60-9.

65. Werch CE, Bian H, Moore MJ, Ames S, DiClemente CC, Weiler RM, et al. Brief multiple behavior interventions in a college student health care clinic. J Adolesc Health. 2007;41:577-85.

66. Yakusheva O, Kapinos K, Weiss M, Yakusheva O, Kapinos K, Weiss M. Peer effects and the freshman 15: evidence from a natural experiment. Econ Hum Biol. 2011;9:119-32.

67. Alpar SE, Senturan L, Karabacak U, Sabuncu N, Alpar SE, Senturan L, et al. Change in the health promoting lifestyle behaviour of Turkish University nursing students from beginning to end of nurse training. Nurse Educ Pract. 2008:8:382-8.
68. Ince ML, Ince ML. Use of a social cognitive theory-based physical-activity intervention on health-promoting behaviors of university students. Percept Mot Skills. 2008;107:833-6.

69. Afifi Soweid R, El Kak F, Major S, Karam D, Rouhana A, Afifi Soweid R, et al. Changes in health-related attitude and self-reported behaviour of undergraduate students at the American university of Beirut following a health awareness course. Educ Health. 2003;16:265-78.

70. Skar S, Sniehotta FF, Molloy GJ, Prestwich A, Araujo-Soares V, Skar S, et al. Do brief online planning interventions increase physical activity amongst university students? A randomised controlled trial. Psychol Health. 2011;26:399-417.

71. Tully MA, Cupples ME. UNISTEP (university students exercise and physical activity) study: a pilot study of the effects of accumulating 10,000 steps on health and fitness among university students. J Phys Act Health. 2011;8:663-7.

72. Huang SJ, Hung WC, Chang M, Chang J, Huang S-J, Hung W-C, et al. The effect of an internet-based, stage-matched message intervention on young Taiwanese women's physical activity. J Health Commun. 2009:14:210-27.

73. Werch CE, Moore MJ, Bian H, DiClemente CC, Ames SC, Weiler RM, et al. Efficacy of a brief image-based multiple-behavior intervention for college students. Ann Behav Med. 2008;36:149-57.

74. Von Ah D, Ebert S, Ngamvitroj A, Park N, Kang DH. Predictors of health behaviours in college students. J Adv Nurs. 2004;48:463-74.

75. Butler SM, Black DR, Blue CL, Gretebeck RJ. Change in diet, physical activity, and body weight in female college freshman. Am J Health Behav. 2004;28:24-32.

76. Elfhag $\mathrm{K}$, Rössner $\mathrm{S}$. Who succeeds in maintaining weight loss? A conceptual review of factors associated with weight loss maintenance and weight regain. Obes Rev. 2005;6:67-85.

\section{Submit your next manuscript to BioMed Central and take full advantage of:}

- Convenient online submission

- Thorough peer review

- No space constraints or color figure charges

- Immediate publication on acceptance

- Inclusion in PubMed, CAS, Scopus and Google Scholar

- Research which is freely available for redistribution 